\title{
Associative retrieval processes in the human medial temporal lobe: Hippocampal retrieval success and CAl mismatch detection
}

\author{
Janice Chen, ${ }^{1,6}$ Rosanna K. Olsen, ${ }^{1,2}$ Alison R. Preston, ${ }^{3}$ Gary H. Glover, ${ }^{4,5}$ and \\ Anthony D. Wagner ${ }^{1,4}$ \\ ${ }^{1}$ Department of Psychology, Stanford University, Stanford, California 94305, USA; ${ }^{2}$ Rotman Research Institute, Toronto, Ontario, M6A \\ 2E1, Canada; ${ }^{3}$ Department of Psychology and Center for Learning \& Memory, University of Texas at Austin, Austin, Texas 78712, USA; \\ ${ }^{4}$ Neurosciences Program, Stanford University, Stanford, California 94305, USA; ${ }^{5}$ Department of Radiology, Stanford University, \\ Stanford, California 94305, USA
}

\begin{abstract}
Hippocampal subfields $C A_{3}$ and $C A_{1}$ are hypothesized to differentially support the generation of associative predictions and the detection of associative mismatches, respectively. Using high-resolution functional MRI, we examined hippocampal subfield activation during associative retrieval and during subsequent comparisons of memory to matching or mismatching decision probes. Activity in the dentate gyrus $/ \mathrm{CA}_{2 / 3}, \mathrm{CA}_{1}$, and other medial temporal lobe subregions tracked associative retrieval success, whereas activity in $\mathrm{CA}_{1}$ and the perirhinal cortex tracked the presence of associative mismatches. These data support the hypothesis that $\mathrm{CA}_{1}$ acts as a "comparator," detecting when memory for the past and sensory input in the present diverge.
\end{abstract}

\begin{abstract}
Although the role of the hippocampus in declarative memory is well established (e.g., Eichenbaum and Cohen 2001), the relative functions of its component subfields remain underspecified. Hippocampal anatomy suggests that subfields $\mathrm{CA}_{3}$ and $\mathrm{CA}_{1}$ differentially support memory-based predictions and the detection of associative novelty, respectively (e.g., Lisman and Grace 2005). Specifically, $\mathrm{CA}_{3}$ is hypothesized to support the encoding of associative memories (Marr 1971; Treves and Rolls 1992; McClelland et al. 1995) that, when later retrieved, act as "predictions" about the present based on knowledge acquired during past events. $\mathrm{CA}_{3}$ predictions are transmitted to $\mathrm{CA}_{1}$, which also receives input from entorhinal cortex regarding the present state of the environment (Jensen and Lisman 1996; Tsodyks et al. 1996). Thus, $\mathrm{CA}_{1}$ may be positioned to act as a "comparator," assessing whether current sensory input matches or mismatches $\mathrm{CA}_{3}$ predictions. $\mathrm{CA}_{1}$ mismatch detection may signal event novelty and foster hippocampal encoding (Hasselmo and Wyble 1997; Vinogradova 2001; Lisman and Grace 2005; Kumaran and Maguire 2007a).

$\mathrm{CA}_{3}$ prediction and $\mathrm{CA}_{1}$ mismatch responses have been explored in animals (e.g., Fyhn et al. 2002; Lee et al. 2004), but limited functional data are available from human $\mathrm{CA}_{3}$ and $\mathrm{CA}_{1}$. In particular, although fMRI studies have revealed increased hippocampal activation during associative novelty (e.g., Kohler et al. 2005; Kumaran and Maguire 2006, 2007b; Duncan et al. 2009; see also, Shohamy and Wagner 2008), the acquired resolution in prior studies was insufficient to distinguish the BOLD response in distinct hippocampal subfields (Carr et al. 2010; although complementary questions have been explored at high resolution, e.g., Bakker et al. 2008; Duncan et al. 2011; Lacy et al. 2011). Accordingly, using high-resolution fMRI (hr-fMRI) (Fig. 1), we examined dentate gyrus $/ \mathrm{CA}_{2 / 3}$ and $\mathrm{CA}_{1}$ function
\end{abstract}

${ }^{6}$ Corresponding author.

E-mail kanile@stanford.edu.

Article is online at http://www.learnmem.org/cgi/doi/10.1101/Im.2135211. during associative retrieval and during the presence of associative novelty (mismatch).

Each of 10 blocks began with a nonscanned encoding phase of 24 face-house pairs presented three times each $(2000 \mathrm{msec}$; 250-msec ISI) in random order. Participants encoded the associations by imagining that each pair constituted a person's face and home. A 1-min distracter phase followed encoding, in which participants viewed left- or right-pointing arrows (1500 msec; 500-msec ISI) and indicated the arrow's direction on each trial by button press. In the subsequent test phase, hr-fMRI data were collected while participants retrieved face-house associations from the most recent encoding phase. Each of 16 test trials began with a fixation cross $(500 \mathrm{msec})$ followed by a face or house cue (1000 msec) and then a 7500-msec delay, during which participants were to covertly recall the other member of the pair (Fig. 2). After the delay, a probe was briefly presented (750 msec followed by a 250 -msec blank screen). On half of the trials the probe was the studied associate of the cue (match); on the other half the probe was a foil drawn from a different study pair (mismatch). The original partners of foils did not appear during test. Participants pressed one of two keys to indicate whether or not the probe was the associate of the cue. Importantly, this decision could not be based merely on stimulus familiarity, as all probes were familiar, differing only in their match/mismatch status. Participants were instructed to respond within $750 \mathrm{msec}$ to encourage recall during the delay. Trials were retained if the reaction time (RT) was $<1000 \mathrm{msec}$. For both match and mismatch conditions, in half of the trials the cue was a house and the probe a face; for the other half the cue was a face and the probe a house (Table 1). Baseline arrow trials were intermixed with test trials, and the order of conditions optimized efficiency of the event-related fMRI design (Dale 1999). Participants gave informed consent, with data from 22 participants submitted to the analysis (data from four others were excluded due to poor behavioral performance [ $d^{\prime}<0.2$ or failure to respond on $>50 \%$ of trials] or $>3 \mathrm{~mm}$ 


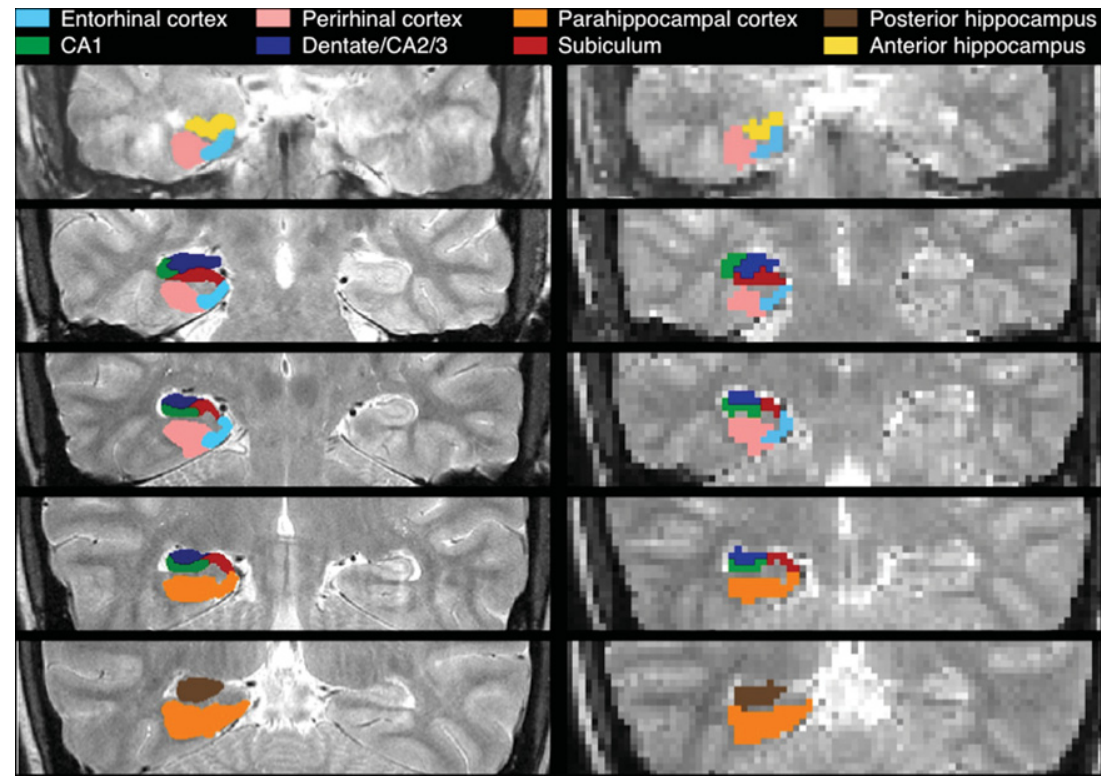

Figure 1. Anatomical ROls. ROls are depicted at anatomical (left, $0.43 \times 0.43 \mathrm{~mm}$ ) and functional (right, $2 \times 2 \mathrm{~mm}$ ) resolution.

motion). For four participants, data from one to two blocks were excluded due to problems with the button box.

MRI data were acquired on a 3.0T GE Signa whole-body system with a receive-only head coil. Structural images (repetition time $[\mathrm{TR}]=3000 \mathrm{msec}$; echo time $[\mathrm{TE}]=68 \mathrm{msec} ; 0.43 \times 0.43 \mathrm{~mm}$ in-plane resolution) and functional images (T2*-sensitive gradient echo spiral in/out pulse sequence [Glover and Law 2001]; $\mathrm{TR}=4000 \mathrm{msec}$; effective $\mathrm{TR}=2000 \mathrm{msec}$; $\mathrm{TE}=34 \mathrm{msec}$; flip angle $=90 ; \mathrm{FOV}=22 \mathrm{~cm} ; 2 \times 2 \mathrm{~mm}$ in-plane resolution) were acquired from 22 3-mm-thick slices perpendicular to the hippocampal axis. Data were slice-time and motion corrected using SPM5 (http://www.fil.ion.ucl.ac.uk/spm/) and custom MATLAB routines; data were neither smoothed nor normalized.

Cued recall and match/mismatch responses were explored in a statistical model treating the cue+delay as an 8500-msec epoch and the probe as an impulse; regressors were convolved with a canonical hemodynamic response function. Cue+delay events for house- and face-cue trials (coded separately) were categorized as correct/incorrect based on response accuracy at probe. Probe events for house- and face-probe trials (coded separately) were categorized based on accuracy and whether the probe was a match or mismatch. The effects of no-response trials (mean $=8.8 \%$ of trials $[\mathrm{SD}=7.7 \%]$, including trials for which $\mathrm{RT}>1000 \mathrm{msec}$ ), RT, motion, block, and linear drift were modeled as regressors of no interest.

Group analyses were performed using anatomically defined region-of-interest (ROI) analyses targeting medial temporal lobe (MTL) subregions (Fig. 1; for details, see Olsen et al. 2009; Preston et al. 2010). For each participant, anatomical ROIs were demarcated on the high-resolution structural images for hippocampal subfields $\left(\mathrm{CA}_{1}\right.$, subiculum, and dentate gyrus/ $\mathrm{CA}_{2 / 3}$; dentate gyrus, $\mathrm{CA}_{2}$, and $\mathrm{CA}_{3}$ cannot be unambiguously segmented at the acquired resolution) and MTL cortical areas (perirhinal [PRc], parahippocampal [PHc], and entorhinal cortex [ERc]), using established procedures (Insausti et al. 1998; Pruessner et al. 2000, 2002; Zeineh et al. 2000, 2003; Insausti and Amaral 2004). To further constrain analyses to voxels that were at least modestly responsive to task demands, functional ROIs were created by defining voxel-level contrast images at a liberal threshold $(t>0.675 ; P<0.25)$ masked with the anatomical ROIs for each participant (see Table 2 for threshold comparisons). For each anatomical ROI and each functional ROI, \% signal change was extracted for each condition, using a deconvolution model to estimate the response at each $\mathrm{TR}$, and then summed over time: effective TRs 3-5 (i.e., 4-10sec post-cue onset) were integrated to capture effects of memory retrieval during the cue+delay (the results reported in the following are qualitatively the same using a 2-8-sec integration window); effective TRs $2-4$ (2-8-sec postprobe onset) were integrated to capture responses to the probe. For each ROI, integrated \% signal change was submitted to repeated-measures analyses of variance (ANOVA), treating subjects as a random effect; hemisphere was included as a factor in all ANOVAs.

Behaviorally, accuracy was higher on mismatch than match trials $\left(F_{(1,21)}=\right.$ 5.55, $P<0.05$; Table 1$)$. Corrected recognition performance (hits-false alarms) neither differed between house-probe and face-probe trials $(P>0.1)$, nor was there a match/ mismatch $\times$ probe-type (house- vs. face-probe) interaction $(P>$ $0.25)$. RTs revealed (1) an effect of accuracy $\left(F_{(1,21)}=12.31, P<\right.$ 0.005 ), with faster RTs on correct trials (match $/$ hits $=657 \mathrm{msec}$; mismatch $/$ correct rejections $=679 \mathrm{msec}$ ) relative to incorrect trials $(\mathrm{match} / \mathrm{misses}=705 \mathrm{msec}$; mismatch $/$ false alarms $=689$ msec) and (2) an effect of probe-type $\left(F_{(1,21)}=17.90, P<0.001\right)$, with faster RTs on face-probe $(666 \mathrm{msec})$ relative to house-probe (699 msec) trials. There was no effect of match $/$ mismatch (match $=681 \mathrm{msec} ;$ mismatch $=684 \mathrm{msec} ; F<1)$, although there was a trend for an accuracy $\times$ match $/$ mismatch interaction $\left(F_{(1,21)}=3.91, P=0.06\right)$, wherein RTs were faster for correct relative to incorrect match trials $\left(F_{(1,21)}=18.74, P<0.001\right)$, but comparable for correct and incorrect mismatch trials $(F<1)$.

To the extent that $\mathrm{CA}_{1}$ supports detection of associative novelty, probe-period activity should depend on the match between the recalled associate and the presented probe-specifically, activity should be greater when the recalled associate and probe
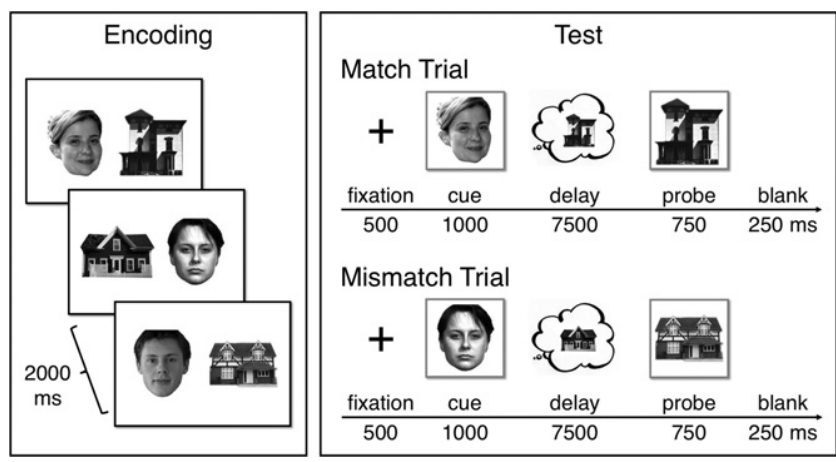

Figure 2. Associative retrieval task. Participants encoded face-house associations. At test, a cue was presented (either a face or a house) followed by a blank delay, during which participants attempted to recall the encoded associate, and then responded to a probe that was either the associate (a match) or a foil (a mismatch). 
Table 1. Percent trials and reaction time for each response type

\begin{tabular}{lccccc}
\hline & \multicolumn{2}{c}{ House-probes } & & \multicolumn{2}{c}{ Face-probes } \\
\cline { 2 - 3 } \cline { 6 - 7 } & $\%($ SD) & RT (SD) & & $\%($ (SD) & RT (SD) \\
\hline Hits & $28.7(7.4)$ & $680(70)$ & & $31.1(7.6)$ & $634(64)$ \\
Correct rejections & $34.4(7.4)$ & $703(51)$ & & $34.4(5.3)$ & $654(59)$ \\
Misses & $16.5(7.7)$ & $718(45)$ & & $15.6(7.4)$ & $693(56)$ \\
False alarms & $10.6(5.9)$ & $694(101)$ & & $11.1(4.1)$ & $684(67)$ \\
No response & $9.8(8.2)$ & - & & $7.8(7.1)$ & - \\
\hline
\end{tabular}

mismatch rather than match. For each anatomical ROI, probe activity for correct match (hit) and mismatch (correct rejection) trials was compared by submitting integrated \% signal change to ANOVA with match/mismatch and probe-type (face- vs. house-probe) included as factors. The effect of match $/$ mismatch was significant in PRc $\left(F_{(1,21)}=4.70, P<0.05\right.$; all other regions $P>0.1$, with greater activity on mismatch than match trials. There also was a match $/$ mismatch $\times$ probe-type interaction in $\mathrm{CA}_{1}\left(F_{(1,21)}=4.42, P<0.05\right.$; all other regions $\left.P>0.1\right)$, due to a mismatch $>$ match pattern in $\mathrm{CA}_{1}\left(F_{(1,21)}=3.86, P=0.06\right)$ that was selective to house-probes (Fig. 3A,B).

To increase sensitivity to putative correlates of associative novelty, functional ROIs restricted analyses to voxels that were at least modestly engaged during the probe period. As a test of the $\mathrm{CA}_{1}$ comparator hypothesis rests on the contrast of correct match vs. mismatch trials, we identified statistically independent task-responsive voxels within each anatomical ROI using the incorrect match trials, i.e., comparison of all misses $>$ baseline. Incorrect mismatch trials were not included during functional ROI definition, because some subjects rarely false alarmed (there were fewer than seven false alarm trials in $\sim 20 \%$ of subjects). From the "probe-responsive" functional ROIs, correct match and mismatch probe-period activity was submitted to ANOVA.

Importantly, $\mathrm{CA}_{1}$ activity was significantly greater for correct mismatch (correct rejections) than match (hits) trials (Fig. 3C,E) $\left(F_{(1,21)}=5.09, P<0.05\right)$, with a trend for an interaction with probe-type $\left(\mathrm{match} / \mathrm{mismatch} \times\right.$ probe-type, $F_{(1,21)}=3.82, P=$ $0.06)$ stemming from significantly greater activity on correct mismatch than match house-probe $\left(F_{(1,21)}=6.05, P<0.05\right)$, but not face-probe $(F<1)$, trials. PRc activity in probe-responsive voxels also demonstrated a trend for a match $/$ mismatch $\times$ probe-type interaction (Fig. 3D,F) $\left(F_{(1,21)}=3.25, P=0.08\right)$. However, in PRc the interaction trend stemmed from a trend for greater activity for correct mismatch than match face-probe $\left(F_{(1,21)}=3.83, P=\right.$ $0.06)$, but not house-probe $(F<1)$, trials. There were no match/ mismatch main effects or interactions in any other region $(P>$ 0.1). Follow-up across-region analyses revealed that the content sensitivity of mismatch enhancement in $\mathrm{CA}_{1}$ and PRc significantly differed (region $\times$ match $/$ mismatch $\times$ probe-type, $F_{(1,21)}=$ 9.95, $P<0.005)$. Thus, consistent with the outcomes of the anatomical ROI analyses, mismatch enhancement effects were seen in $\mathrm{CA}_{1}$ and PRc voxels that were independently identified as functionally engaged during the probe period, with these analyses further suggesting across-region differential sensitivity to probe-type (although inferences about content sensitivity should be treated with caution, as match/mismatch $\times$ probe-type interactions were trends in both $\mathrm{CA}_{1}$ and $\mathrm{PRc}$ ).

Turning to the second question of interest-whether $\mathrm{CA}_{3}$ activity is related the upcoming Figure $3 \mathrm{C}, \mathrm{D}$. to associative retrieval success-we investigated cued recall effects by analyzing activity during the cue+delay period, when participants viewed a face- or house-cue and attempted to retrieve the associate. For each anatomical ROI, integrated \% signal change was submitted to ANOVA, including factors of accuracy and cuetype (face- or house-cue). A main effect of accuracy was observed in multiple MTL subregions (Fig. 4), with activity being greater during correct than incorrect retrieval trials in dentate gyrus/ $\mathrm{CA}_{2 / 3}\left(F_{(1,21)}=6.19, P<0.05\right), \mathrm{CA}_{1}\left(F_{(1,21)}=12.28, P<0.005\right)$, subiculum $\left(F_{(1,21)}=4.42, \quad P<0.05\right)$, posterior hippocampus $\left(F_{(1,21)}=5.34, P<0.05\right)$, PHc $\left(F_{(1,21)}=19.27, P<0.0005\right)$, and PRc $\left(F_{(1,21)}=6.84, P<0.05\right)$. PHc also demonstrated an effect of cue-type $\left(F_{(1,21)}=13.93, P=0.001\right)$, with a greater response to house-cues, and a hemisphere $\times$ cue-type interaction $\left(F_{(1,21)}=\right.$ 26.97, $P<0.001$ ), with the house- $>$ face-cue difference being greater in the left hemisphere. Collectively, the cue+delay period data revealed that the BOLD response in multiple MTL regions was greater prior to accurate than inaccurate speeded associative recognition decisions.

The present hr-fMRI data provide novel evidence regarding the functional properties of human dentate gyrus/ $\mathrm{CA}_{2 / 3}$ and $\mathrm{CA}_{1}$, and further document mnemonic and stimulus class effects in hippocampal and MTL cortical subregions. There were two main findings. First, activity during the decision probe was greater in $\mathrm{CA}_{1}$ and PRc when the participant correctly recalled the learned associate and the recognition probe differed from (mismatched) this retrieved memory, a finding consistent with the hypothesis that $\mathrm{CA}_{1}$ acts as a comparator, differentiating between perceptual inputs that are mnemonically unexpected vs. expected (e.g., Lisman and Grace 2005; for related hr-fMRI data, see Duncan et al. 2011). In this framework, mismatches may constitute associative "prediction errors" between expected and actual events, analogous to those described in models of reinforcement learning (Rescorla and Wagner 1972). In such models, unexpected rewards and punishments yield more learning than expected rewards and punishments. Within the hippocampal learning system, memory retrieval can be thought of as an act of prediction in which the level of expectation varies according to the strength of the memory; repetition is by definition expected while novelty is unexpected. Thus, hippocampal "prediction error" describes the detection of an unexpected event (associative novelty) which may serve to improve learning. This "prediction error" signal may operate by driving "reward" responses in ventral striatum that, in turn, trigger the up-regulation of midbrain dopamine release that ultimately enhances hippocampal learning (Lisman and Grace 2005). Hippocampal prediction error may occur at the "item level" (i.e., when an associative memory predicts the occurrence of a specific item, but the prediction is violated) or the "context level" (i.e., when predictions about the relative probabilities of outcomes in a given context are violated) (e.g., Summerfield et al. 2008).

Table 2. Mismatch effects in probe-responsive voxels

\begin{tabular}{lcccc} 
Voxel bin & $\mathrm{CA}_{\mathbf{1}}$ house-probes & $\mathrm{CA}_{\mathbf{1}}$ face-probes & PRc house-probes & PRc face-probes \\
\hline$T>0.675$ & $0.32(P<0.05)$ & -0.02 & 0.00 & $0.45(P<0.05)$ \\
$0<T<0.675$ & $0.24(P=0.05)$ & -0.01 & -0.10 & $0.36(P<0.05)$ \\
$-0.675<T<0$ & $0.26(P=0.12)$ & -0.05 & 0.13 & $0.26(P=0.10)$ \\
$T<-0.675$ & $0.03(P>0.8)$ & -0.05 & 0.04 & $0.28(P>0.2)$ \\
\hline
\end{tabular}

Mismatch effects (correct rejections - hits in $\mathrm{CA}_{1}$ house-probes and PRc face-probes) were significant in any voxels where $T>0$ in the independent contrast of misses $>$ baseline, suggesting that even voxels only weakly above baseline during miss trials may nonetheless contribute to associative novelty responses. No significant mismatch effects were found in $\mathrm{CA}_{1}$ face-probes or PRc house-probes. First row corresponds to 

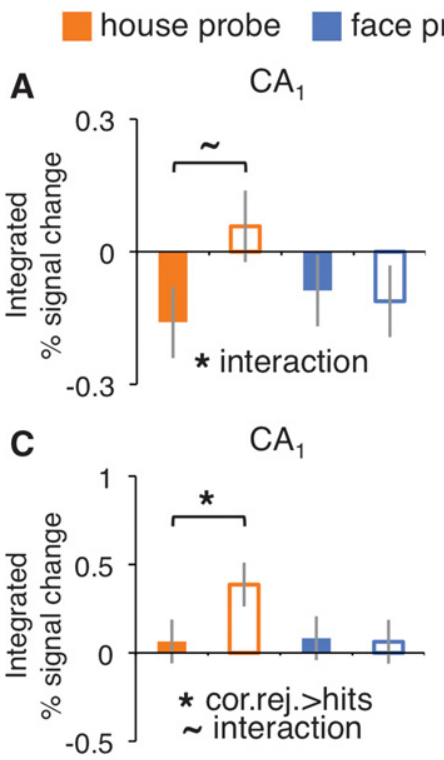

D
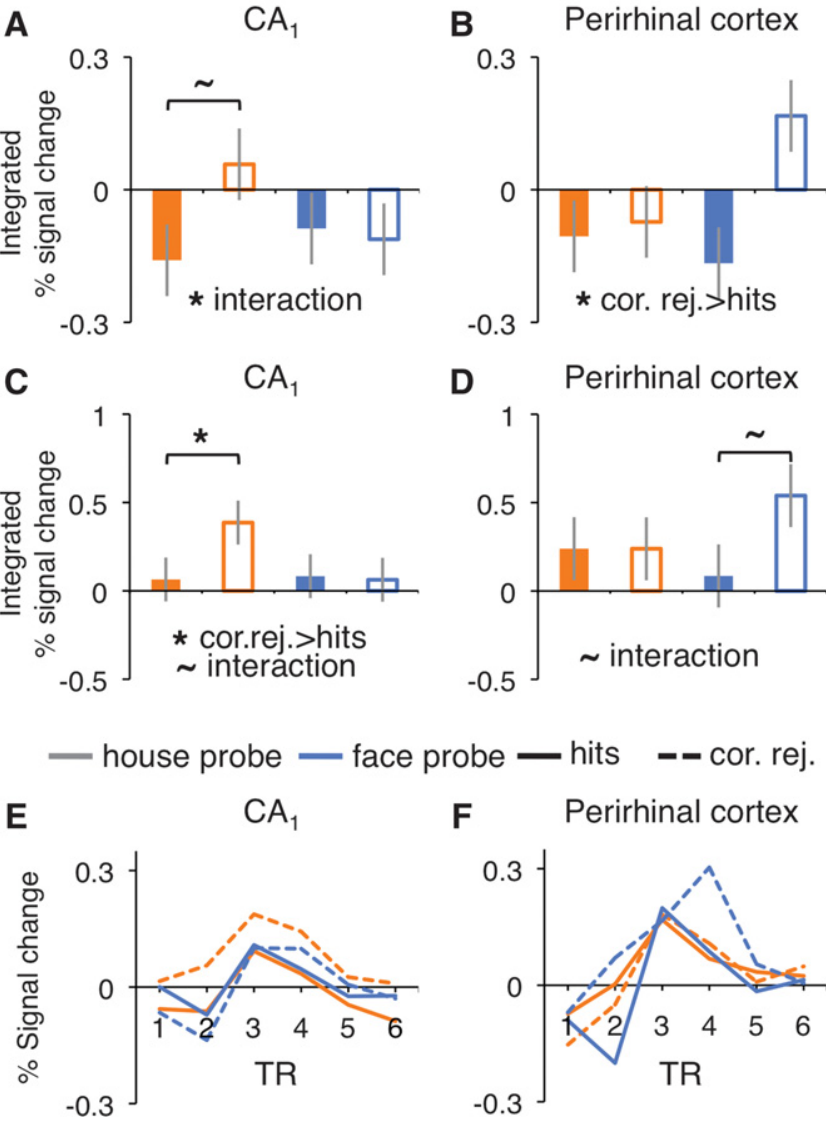

Figure 3. Associative novelty (correct rejections $>$ hits). BOLD response during probe period, integrated over $2-8 \mathrm{sec}$ (TRs 2-4) after stimulus onset, from all $C_{1}$ voxels $(A)$, all PRc voxels $(B)$, and probe-responsive voxels $(C, D)$. Time course of BOLD response during probe period from probe-responsive voxels in $\mathrm{CA}_{1}(E)$ and $\mathrm{PRC}(F) .{ }^{*} P<0.05, \sim P<0.10$.

Interestingly, activation in $\mathrm{CA}_{1}$ was greater when participants encountered house-probes that violated their mnemonic predictions (correct mismatch) relative to probes that confirmed these predictions (correct match), whereas PRc activation tended to show such an effect when participants encountered faceprobes; the pattern of mismatch enhancement significantly differed by content across these two regions. The content-sensitive nature of these effects possibly reflects interactions between associative novelty and our selected stimuli; that is, differential responses due to (1) inherent stimulus category preferences (e.g., differential sensitivity for spatial/house stimuli), (2) greater task difficulty-e.g., higher intraclass perceptual variability-for one set relative to the other (as suggested by the observed reaction times), or (3) some combination of these factors. Further research is needed to determine whether stimulus class systematically influences mismatch responses in the MTL.

In contrast to the present observation of a $\mathrm{CA}_{1}$ associative novelty (mismatch enhancement) effect, several prior fMRI studies have revealed hippocampal match enhancement. Hannula and Ranganath (2008) observed greater hippocampal activation when the probe matched an imagined multiobject configuration. Similarly, Duncan et al. (2009) observed greater hippocampal activity when the probe matched an imagined display, but greater activation on mismatch trials when the probe contained unexpected perceptual novelty. The first pattern was interpreted as a goal-modulated match signal, and the second as an automatic mismatch signal (for related $\mathrm{CA}_{1}$ data, see Duncan et al. 2011). By contrast, Dudukovic et al. (2011) proposed that match enhancement occurs when a previously encountered stimulus (e.g., a decision probe) triggers the retrieval of other event details that co-occurred with the stimulus's previous encounter. From this perspective, probe-triggered pattern completion processes can mask the presence of mismatch effects in the BOLD signal. The present data are broadly consistent with this latter account, as participants in the present experiment were encouraged to (1) "pattern complete" (retrieve) event details during the cue + delay period and (2) make rapid decisions when presented with the probe, both of which were intended to minimize the amount of retrieval occurring at the probe stage. The design temporally segregated retrieval and comparison demands, permitting detection of $\mathrm{CA}_{1}$ mismatch enhancement during the probe stage. One potential drawback of this temporal segregation is that it requires associative information to be maintained across a long delay, potentially making the mapping to comparator models challenging due to differing timescales. Although previous work has shown that hippocampal subregions are recruited during maintenance of information prior to a match $/$ mismatch decision (Olsen et al. 2009), future work is required to understand the influence of such delays on the computation and magnitude of $\mathrm{CA}_{1}$ mismatch effects. Moreover, given that prediction error signals are posited to foster the encoding of unexpected events, future research, potentially utilizing a subsequent memory test, is needed to examine whether dentate $/ \mathrm{CA}_{2 / 3}$ mediates new associative encoding as a consequence of $\mathrm{CA}_{1}$ prediction error detection.

Our second main finding was that activity during the cue+ delay period in dentate gyrus $/ \mathrm{CA}_{2 / 3}$ and $\mathrm{CA}_{1}$, along with other MTL subregions, varied according to subsequent accuracy on the decision probe, consistent with the hypothesis that $\mathrm{CA}_{3}$ supports associative retrieval (e.g., Norman and O'Reilly 2003). This pattern is also compatible with previous reports that the effects of associative retrieval propagate to other hippocampal and cortical structures (e.g., Naya et al. 2001), though it is important to

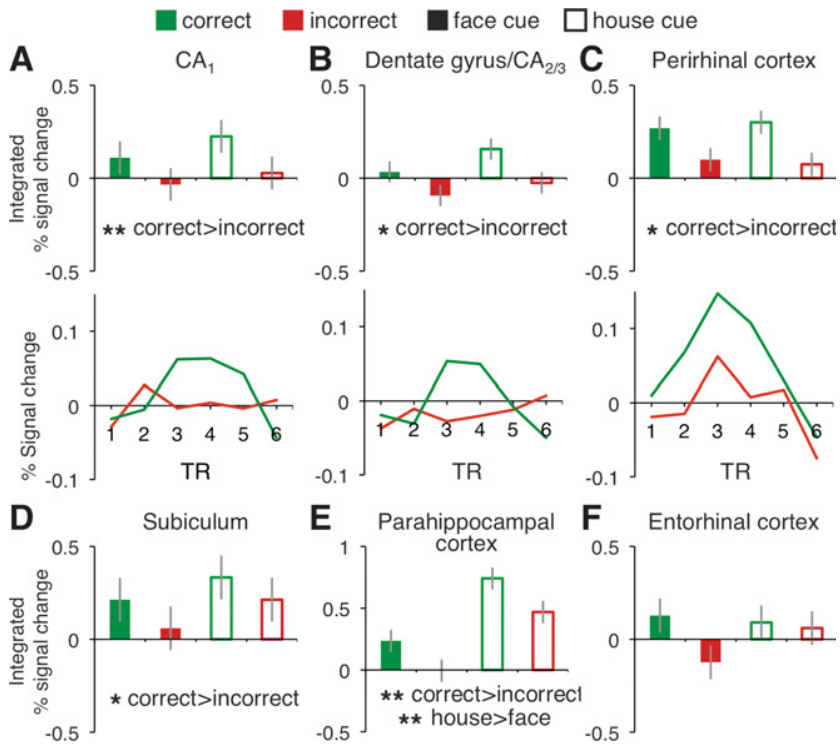

Figure 4. Cued recall. BOLD response in each anatomically defined MTL region during the cue+delay period, integrated over 4-10 sec (TRs 3-5) after stimulus onset $(A-F)$, with accompanying time courses (face- and house-cues combined) for $\mathrm{CA}_{1}$, dentate gyrus $/ \mathrm{CA}_{2 / 3}$, and $\operatorname{PRc}(A-C) .{ }^{*} P<0.05,{ }^{* * P}<0.005$. 
note that the direction of signal propagation cannot be determined in the current study due to the coarse temporal resolution of the BOLD signal, and thus our results do not preclude the possibility that retrieval effects could originate in different hippocampal and MTL subregions independently. These data extend prior fMRI studies that documented associative retrieval effects in human hippocampus (e.g., Small et al. 2001; Cansino et al. 2002; Dobbins et al. 2003; Zeineh et al. 2003; Preston et al. 2004; Ranganath et al. 2004; Eldridge et al. 2005; Meltzer and Constable 2005; Hannula and Ranganath 2009; Ross et al. 2009). In the present data, cue+delay period BOLD activity in hippocampus and MTL cortex predicted participants' accuracy, moments later, at identifying whether a probe was previously paired with the cue. This effect likely reflects the successful retrieval of the encoded associate, supporting an accurate memory-guided decision. Our data also extend recent hr-fMRI data documenting retrieval effects in subiculum (e.g., Zeineh et al. 2003; Eldridge et al. 2005). However, in contrast to these earlier reports, here retrieval success also modulated cue+delay period activity in both dentate gyrus $/ \mathrm{CA}_{2 / 3}$ and $\mathrm{CA}_{1}$ (as well as in other MTL structures). As such, the present data challenge the hypothesis that intrahippocampal functional differentiation is organized according to encoding vs. retrieval processes (see also, Preston et al. 2010).

In summary, the hippocampus may continuously predict that the present will resemble the past and concurrently monitor for when predictions are violated, fostering differential encoding of the unexpected (e.g., Lisman and Grace 2005; Kumaran and Maguire 2009). Our findings highlight a role for $\mathrm{CA}_{1}$ in signaling when the present deviates from the past.

\section{Acknowledgments}

Supported by the National Institute of Mental Health (5R01MH076932), the National Alliance for Research on Schizophrenia and Depression, and the Alfred P. Sloan Foundation.

\section{References}

Bakker A, Kirwan CB, Miller M, Stark CEL. 2008. Pattern separation in the human hippocampal CA3 and dentate gyrus. Science 319: 1640-1642.

Cansino S, Maquet P, Dolan RJ, Rugg MD. 2002. Brain activity underlying encoding and retrieval of source memory. Cereb Cortex 12: $1048-1056$

Carr VA, Rissman J, Wagner AD. 2010. Imaging the human medial temporal lobe with high-resolution fMRI. Neuron 65: 298-308.

Dale AM. 1999. Optimal experimental design for event-related fMRI. Hum Brain Mapp 8: 109-114.

Dobbins IG, Rice HJ, Wagner AD, Schacter DL. 2003. Memory orientation and success: Separable neurocognitive components underlying episodic recognition. Neuropsychologia 41: 318-333.

Dudukovic NM, Preston AR, Archie JJ, Glover GH, Wagner AD. 2011. High-resolution fMRI reveals match enhancement and attentional modulation in the human medial temporal lobe. J Cogn Neurosci 23: $670-682$.

Duncan K, Curtis C, Davachi L. 2009. Distinct memory signatures in the hippocampus: Intentional states distinguish match and mismatch enhancement signals. J Neurosci 29: 131-139.

Duncan K, Ketz N, Inata SJ, Davachi L. 2011. Evidence for area CA1 as a match/mismatch detector: A high-resolution fMRI study of the human hippocampus. Hippocampus (in press).

Eichenbaum H, Cohen NJ. 2001. From conditioning to conscious recollection: Memory systems of the brain. Oxford University Press, New York.

Eldridge LL, Engel SA, Zeineh MM, Bookheimer SY, Knowlton BJ. 2005. A dissociation of encoding and retrieval processes in the human hippocampus. J Neurosci 25: 3280-3286.

Fyhn M, Molden S, Hollup S, Moser M-B, Moser E. 2002. Hippocampal neurons responding to first-time dislocation of a target object. Neuron 35: $555-566$.

Glover GH, Law CS. 2001. Spiral-in/out BOLD fMRI for increased SNR and reduced susceptibility artifacts. Magn Reson Med 46: 515-522.

Hannula DE, Ranganath C. 2008. Medial temporal lobe activity predicts successful relational memory binding. J Neurosci 28: 116-124.
Hannula DE, Ranganath C. 2009. The eyes have it: Hippocampal activity predicts expression of memory in eye movements. Neuron 63: 592-599.

Hasselmo ME, Wyble BP. 1997. Free recall and recognition in a network model of the hippocampus: Simulating effects of scopolamine on human memory function. Behav Brain Res 89: 1-34.

Insausti R, Amaral DG. 2004. Hippocampal formation. In The human nervous system (ed. G Paxinos, KM Jurgen), pp. 871-913. Elsevier Academic, San Diego, CA.

Insausti R, Juottonen K, Soininen H, Insausti AM, Partanen K, Vainio P, Laakso MP, Pitkanen A. 1998. MR volumetric analysis of the human entorhinal, perirhinal, and temporopolar cortices. Am J Neuroradiol 19: $659-671$.

Jensen O, Lisman JE. 1996. Hippocampal CA3 region predicts memory sequences: Accounting for the phase precession of place cells. Learn Mem 3: 279-287.

Kohler S, Danckert S, Gati JS, Menon RS. 2005. Novelty responses to relational and non-relational information in the hippocampus and the parahippocampal region: A comparison based on event-related fMRI. Hippocampus 15: $763-774$.

Kumaran D, Maguire EA. 2006. An unexpected sequence of events: Mismatch detection in the human hippocampus. PLoS Biol 4: e424. 10.1371/journal.pbio.0040424.

Kumaran D, Maguire EA. 2007a. Which computational mechanisms operate in the hippocampus during novelty detection? Hippocampus 17: $735-748$

Kumaran D, Maguire EA. 2007b. Match mismatch processes underlie human hippocampal responses to associative novelty. J Neurosci 27: $8517-8524$.

Kumaran D, Maguire EA. 2009. Novelty signals: A window into hippocampal information processing. Trends Cogn Sci 13: 47-54.

Lacy JW, Yassa MA, Stark SM, Muftuler LT, Stark CEL. 2011. Distinct pattern separation related transfer functions in human CA3/dentate and CA1 revealed using high-resolution fMRI and variable mnemonic similarity. Learn Mem 18: 15-18.

Lee I, Yoganarasimha D, Rao G, Knierim JJ. 2004. Comparison of population coherence of place cells in hippocampal subfields CA1 and CA3. Nature 430: $456-459$.

Lisman JE, Grace AA. 2005. The hippocampal-VTA loop: Controlling the entry of information into long-term memory. Neuron 46: 703-713.

Marr D. 1971. Simple memory: A theory for archicortex. Philos Trans R Soc Lond B Biol Sci 262: 23-81.

McClelland JL, McNaughton BL, O'Reilly RC. 1995. Why there are complementary learning systems in the hippocampus and neocortex: Insights from the successes and failures of connectionist models of learning and memory. Psych Rev 102: 419-457.

Meltzer JA, Constable RT. 2005. Activation of human hippocampal formation reflects success in both encoding and cued recall of paired associates. Neuroimage 24: 384-397.

Naya Y, Yoshida M, Miyashita Y. 2001. Backward spreading of memoryretrieval signal in the primate temporal cortex. Science 291: 661-664.

Norman KA, O'Reilly RC. 2003. Modeling hippocampal and neocortical contributions to recognition memory: A complementary-learningsystems approach. Psychol Rev 110: 611-646.

Olsen RK, Nichols EA, Chen J, Hunt JF, Glover GH, Gabrieli JD, Wagner AD. 2009. Performance-related sustained and anticipatory activity in human medial temporal lobe during delayed match-to-sample. $J$ Neurosci 29: 11880-11890.

Preston AR, Shrager Y, Dudukovic NM, Gabrieli JD. 2004. Hippocampal contribution to the novel use of relational information in declarative memory. Hippocampus 14: 148-152.

Preston AR, Bornstein AM, Hutchinson JB, Gaare ME, Glover GH, Wagner AD. 2010. High-resolution fMRI of content-sensitive subsequent memory responses in human medial temporal lobe. J Cogn Neurosci 22: $156-173$.

Pruessner JC, Li LM, Serles W, Pruessner M, Collins DL, Kabani N, Lupien S, Evans AC. 2000. Volumetry of hippocampus and amygdala with high-resolution MRI and three-dimensional analysis software: Minimizing the discrepancies between laboratories. Cereb Cortex 10: $433-442$

Pruessner JC, Kohler S, Crane J, Pruessner M, Lord C, Byrne A, Kabani N, Collins DL, Evans AC. 2002. Volumetry of temporopolar, perirhinal, entorhinal and parahippocampal cortex from high-resolution MR images: Considering the variability of the collateral sulcus. Cereb Cortex 12: $1342-1353$.

Ranganath C, Cohen MX, Dam C, D’Esposito M. 2004. Inferior temporal, prefrontal, and hippocampal contributions to visual working memory maintenance and associative memory retrieval. J Neurosci 24: 3917 3925.

Rescorla RA, Wagner AR. 1972. A theory of Pavlovian conditioning: Variations in the effectiveness of reinforcement and nonreinforcement. In Classical conditioning II (ed. AH Black, WF Prokasy), pp. 64-88. Appleton-Century-Crofts, New York. 
Ross RS, Brown TI, Stern CE. 2009. The retrieval of learned sequences engages the hippocampus: Evidence from fMRI. Hippocampus 19: 790-799.

Shohamy D, Wagner AD. 2008. Integrating memories in the human brain: Hippocampal-midbrain encoding of overlapping events. Neuron 60: 378-389.

Small SA, Nava AS, Perera GM, DeLaPaz R, Mayeux R, Stern Y. 2001. Circuit mechanisms underlying memory encoding and retrieval in the long axis of the hippocampal formation. Nat Neurosci 4: 442-449.

Summerfield C, Trittschuh EH, Monti JM, Mesulam MM, Egner T. 2008.

Neural repetition suppression reflects fulfilled perceptual expectations. Nat Neurosci 11: 1004-1006.

Treves A, Rolls ET. 1992. Computational constraints suggest the need for two distinct input systems to the hippocampal CA3 network. Hippocampus 2: 189-199.
Tsodyks MV, Skaggs WE, Sejnowski TJ, McNaughton BL. 1996. Population dynamics and theta rhythm phase precession of hippocampal place cell firing: A spiking neuron model. Hippocampus 6: 271-280.

Vinogradova OS. 2001. Hippocampus as comparator: Role of the two input and two output systems of the hippocampus in selection and registration of information. Hippocampus 11: 578-598.

Zeineh MM, Engel SA, Bookheimer SY. 2000. Application of cortical unfolding techniques to functional MRI of the human hippocampal region. NeuroImage 11: 668-683.

Zeineh MM, Engel SA, Thompson PM, Bookheimer SY. 2003. Dynamics of the hippocampus during encoding and retrieval of face-name pairs. Science 299: 577-580.

Received January 5, 2011; accepted in revised form June 8, 2011. 


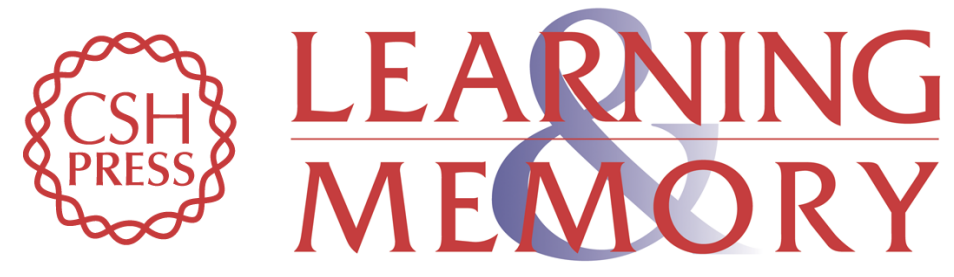

\section{Associative retrieval processes in the human medial temporal lobe: Hippocampal retrieval success and CA1 mismatch detection}

Janice Chen, Rosanna K. Olsen, Alison R. Preston, et al.

Learn. Mem. 2011, 18:

Access the most recent version at doi:10.1101//m.2135211

References This article cites 43 articles, 10 of which can be accessed free at:

http://learnmem.cshlp.org/content/18/8/523.full.html\#ref-list-1

License

Email Alerting Receive free email alerts when new articles cite this article - sign up in the box at the Service top right corner of the article or click here. 